\title{
El cigarrillo electrónico: Peligro u oportunidad
}

\author{
Sergio Monraz-Pérez, Justino Regalado-Pineda, Rogelio Pérez-Padilla $\bowtie$
}

Instituto Nacional de Enfermedades Respiratorias Ismael Cosío Villegas, Ciudad de México.

Trabajo recibido: 03-VI-2015; aceptado: 03-VI-2015

\section{INTRODUCCIÓN}

Los cigarrillos electrónicos (CE) fueron inventados en China, entraron al mercado en 2003 y su uso se ha ido extendiendo rápidamente por todo el mundo con o sin autorización oficial gracias a una intensa publicidad y al indudable ingenio del aparato. Se crearon para reproducir la experiencia de fumar incluyendo el manejo entre los dedos, la inhalación y la exhalación de un vapor generado por un atomizador a partir de un cartucho líquido (de contenido variable), o bien de una cámara de vaporización mediante calentamiento electrónico por una batería que se activa por la inhalación o al presionar un botón. ${ }^{1,2}$

\section{EPIDEMIOLOGÍA}

En Estados Unidos su uso al menos ocasional casi se triplicó entre 2010 y 2013 (de 3.3 a 8.5\%), ${ }^{3}$ y su uso habitual pasó de 0.3 al $6.8 \%,{ }^{4}$ incluyendo a adolescentes. ${ }^{5}$ En el año 2013, 3\% de los alumnos de secundaria en Estados Unidos y $11.9 \%$ de los alumnos de preparatoria refirieron haber utilizado al menos una vez un CE, comparado con 1.1 y $4.5 \%$, respectivamente que lo utilizaron en los 30 días previos a la encuesta. ${ }^{6}$

Afortunadamente este incremento en el uso del CE se hace primordialmente a expensas del cigarrillo convencional ${ }^{7}$ ya que la mayoría de los fumadores activos de CE fumaban previamente cigarrillos convencionales ${ }^{8,9}$ y sólo el $1.2 \%$ de los nunca fumadores de Estados Unidos y $0.5 \%$ de los nunca fumadores en Inglaterra probaron inicialmente el CE. 9,10 En una encuesta realizada por Internet en Estados Unidos, entre el año 2010 y 2013, el uso de CE aumentó entre los fumadores activos (de 9.8 a $36.5 \%$ ), y fumadores previos (2.5 a $9.6 \%)$, pero no se modificó en los nunca fumadores (1.3 a $1.2 \%)^{9}$

A pesar de esto, preocupa el atractivo hacia los jóvenes y su potencial adictivo y posterior transición hacia el cigarrillo convencional, ${ }^{11-13}$ y en este sentido, en una encuesta nacional de estudiantes de secundaria en Estados Unidos, se mostró que el $44 \%$ de los que alguna vez probaron el CE (escasos como se describió en el párrafo previo), probaron posteriormente el cigarrillo convencional,${ }^{14}$ y queda la duda si puede ser puerta de entrada al uso de otras drogas ilegales, ${ }^{12}$ como se ha considerado al cigarrillo convencional. Los fumadores de $C E$, hombres y mujeres por igual, suelen ser más jóvenes, con mayor nivel socioeconómico y educativo que los de cigarrillos convencionales..$^{15-17}$ Por lo regular no son fumadores intensos y aproximadamente 12-14\% de los fumadores que prueban los CE continúan con su uso diario. ${ }^{8}$ En México y Latinoamérica se carece hasta el momento de información similar.

\section{DISPOSITIVO, CONTENIDO Y COMPARACIONES}

Para el año 2014 había alrededor de 466 marcas de CE y más de 7,000 saborizantes, por otro lado, el CE se ha sofisticado tecnológicamente incorporando sensor de flujo, control de calentamiento del dispositivo, diodo para emitir luz, baterías recargables, cartuchos con descarga de concentraciones altas de nicotina y posibilidad de reúso. ${ }^{1,2}$ Los cartuchos pueden tener o no nicotina, pero la pureza no es tan alta como en los substitutos médicos de la nicotina, ya que se han identificado algunos componentes con potencial carcinógeno comunes en el cigarrillo convencional y que demanda demostrar su seguridad a largo plazo. ${ }^{18-22} \mathrm{El}$ contenido de nicotina declarado en los CE va desde 0 (libre de nicotina) hasta $100 \mathrm{mg} / \mathrm{mL}^{23}$ y produce niveles medibles de cotinina en sangre, pero en el análisis químico independiente han encontrado cantidades que difieren de la señalada ${ }^{24} \mathrm{e}$ inclusive han encontrado nicotina en algunos cartuchos reportados como «libres de nicotina». ${ }^{19,25}$ La cantidad de nicotina entregada por el dispositivo depende de la concentración de nicotina en el líquido, la intensidad de la inspiración, y otras características dependientes 
del dispositivo. ${ }^{26}$ Otros componentes incluyen conservadores como propilenglicol, glicerol, etilenglicol 27,28 y cantidades medibles reportadas de estaño, plomo, níquel, cromo, nitrosaminas, compuestos carbamilo, metales, compuestos orgánicos volátiles, y compuestos fenólicos. ${ }^{18-20,22,24,29-31}$ El costo del dispositivo es variable y su mantenimiento incluye la compra de cartuchos, baterías, cargadores y la frecuencia de uso. En total, el gasto regular para un fumador puede superar al de los cigarrillos convencionales.

Varios de los componentes del CE incluyendo saborizantes, generan irritantes y/o carcinógenos (clasificados por la Agencia Internacional de Investigación en Cáncer) como el formaldehído (grupo 1), acetaldehído y óxido de propileno (grupo 2B), la acroleína (toxina) del glicerol, nitrosaminas, metales, compuestos orgánicos volátiles, carbonilo y fenólicos. ${ }^{11-13,32-34}$ Sin embargo, se encuentran en cantidades menores en comparación con el cigarrillo convencional. 18-20,22,30,31 El uso de CE en 30 adultos ocasionó aumento ligero en la resistencia al flujo e impedancia, y disminución de la fracción exhalada de óxido nítrico en el grupo experimental comparado con placebo. ${ }^{35}$ La nicotina, además de ser altamente adictiva, puede ser letal en cantidades pequeñas (0.5-1 $\mathrm{mg} / \mathrm{kg}$ de peso en adultos y 0.1-0.2 $\mathrm{mg} / \mathrm{kg}$ de peso en niños), peligroso en los niños que podrían inhalar, ingerir o incluso al tener contacto con la piel de forma accidental con el contenido de los cartuchos. En Estados Unidos ha incrementado paulatinamente el número de reportes por intoxicación relacionadas al CE de 1 por mes en el 2010 hasta 215 por mes en $2014 .^{36}$

\section{POSICIÓN DE ORGANIZACIONES MÉDICAS Y DE LA ORGANIZACIÓN MUNDIAL DE LA SALUD}

Múltiples organizaciones y sociedades médicas han elaborado recomendaciones y su posición respecto al uso de CE, entre éstas la Asociación Americana del Corazón, ${ }^{1}$ la Sociedad Española de Neumología y Cirugía Torácica (SEPAR), ${ }^{37}$ facilitando a la FDA (del inglés Food and Drug Administration) la regulación de los productos derivados del tabaco y el CE. ${ }^{1}$ El Foro Internacional de Sociedades Respiratorias (FIRS) insiste en que los riesgos a la salud de los CE aún no han sido evaluados de forma adecuada, ni el potencial adictivo de la nicotina; de momento se carece de suficientes estudios sobre el potencial benéfico de los CE como terapia de sustitución de nicotina, así como sobre el posible daño ante la exposición pasiva a los vapores, por lo que aconsejan restringir su uso hasta que se tenga esta información. También sugieren que en caso de que se llegue a permitir su uso, éste debería de ser regulado (como medicamentos o en su defecto como productos del tabaco) y recomiendan impulsar la investigación de los efectos de la salud de estos dispositivos electrónicos y monitorizar en la población los patrones de uso y consecuencias de los dispositivos electrónicos. ${ }^{23}$ Además, se ha recomendado restringir la publicidad y propaganda de los $\mathrm{CE}$, como en el caso de los cigarrillos convencionales.

La Organización Mundial de la Salud (OMS) ha fijado su posición con relación al $\mathrm{CE}^{38,39}$ y recomienda: a) impedir la promoción del CE entre no fumadores y población joven para minimizar los riesgo potenciales para la salud entre los usuarios del CE, así como entre la población general; b) prohibir la difusión de resultados no comprobados de posibles efectos saludables derivados del uso del CE y, principalmente, c) proteger las políticas públicas de control de tabaco vigentes de los intereses comerciales impulsados por la industria tabacalera para promover y regularizar el uso del CE. El informe abunda sobre la necesidad de los países de regular apropiadamente la publicidad, promoción y patrocinio de los CE, así como la necesidad de regular $y$, de hecho, prohibir el uso de este tipo de dispositivos en espacios cerrados donde actualmente está prohibido fumar.

\section{LEGISLACIÓN EN MÉXICO Y EN EL MUNDO}

La FDA no ha aprobado a los CE como herramienta para dejar de fumar. Una de las principales medidas por regular es el registro de los productos y mostrar con precisión (con un adecuado etiquetado) los ingredientes que contienen, además de prohibir su venta a menores de edad..$^{40}$ En Estados Unidos aún no se ha regularizado la venta de CE y están disponibles para su venta sin restricción de la edad; además de que pueden conseguirse por Internet, carecen de advertencias visuales en el empaque y de regulación respecto al contenido del líquido del cartucho. ${ }^{41-43}$ En México, la importación, distribución, comercialización y venta del denominado CE está prohibida aún por Internet a través de la Ley General para el Control del Tabaco, y se ha solicitado a las secretarías de salud de los estados vigilar el cumplimiento de esta legislación. ${ }^{44}$

A pesar de que la venta de cigarrillos electrónicos está prohibida en muchos países (México, Australia, Brasil, Canadá, Panamá, Singapur, Suiza), no es universal como se demuestra por la situación de Estados Unidos. ${ }^{45}$

\section{DISCUSIÓN}

La oposición al CE ha sido feroz por muchos grupos antitabaco, resaltando apropiadamente la falta de 
resultados de seguridad a largo plazo, el potencial adictivo de la nicotina, y mostrando el pánico a que los adolescentes se inicien en la adicción por su uso y a la postre usen los cigarrillos convencionales. Hasta ahora, la experiencia habla de que si bien esto llega a suceder, la inmensa mayoría de los usuarios del CE eran fumadores activos o exfumadores. Es decir, que en la práctica está usándose como instrumento para dejar de fumar, similar a la de un sustituto de nicotina que simula más la experiencia de fumar.

En otro extremo está la posición de los que consideran que la toxicidad esperada de estos dispositivos será substancialmente menor a la del cigarrillo convencional ya que no se genera una combustión ni la liberación de miles de tóxicos en cantidades gigantescas como sucede al fumar. Esta transición generaría un rescate de muchas vidas y reducción en la incapacidad. El uso directo del tabaco genera 6 millones de muertes al año y el tabaquismo pasivo 500,000; conjuntamente, muchos años de vida saludable perdidos e incapacidad, y no es un asunto despreciable aprovechar todos los recursos posibles para reducir esta carga de enfermedad.

El costo de los sustitutos de nicotina es alto y algunos otros medicamentos utilizados para dejar de fumar como el bupropión y la vareniclina, además del costo elevado, pueden tener efectos adversos significativos. Si bien, es importante que se conozca la toxicidad a mediano y largo plazo y se tomen decisiones comparando el CE y el cigarrillo convencional, muchas personas con problemas graves por fumar no tolerarían esta espera, y podrían desperdiciar la oportunidad de cambiar a un producto menos tóxico si no pueden suspender por completo el hábito tabáquico. Como instrumento de inicio de la adicción a la nicotina sin duda preocupa, pero su condición debería ser ilegal para los menores así como es ilegal la compra de cigarrillos, pero desgraciadamente la venta a menores de cigarrillos convencionales o electrónicos sigue sucediendo en todo el mundo.

En el grupo que inicia fumando el CE es esperable que la nicotina genere más efectos adversos; aunque no es esperable esto en fumadores crónicos que ya se encuentran acostumbrados a la misma. Se han descrito en los ensayos clínicos más efectos adversos a los cigarrillos electrónicos que a los sustitutos de nicotina aprobados, ${ }^{46}$ lo que pudiera ser producto de comparar dosis diferentes dada la heterogeneidad en el contenido de nicotina de los CE. En un reciente metaanálisis ${ }^{47}$ en el que se incluyeron seis estudios del uso de CE para dejar de fumar, se observó buena efectividad del CE con nicotina vs. sin nicotina para el abandono del hábito tabáquico, además de observar reducción del número de cigarrillos fumados diariamente. En este metaanálisis se concluye que en caso de demostrar la seguridad de los $C E$, podrían ser de gran utilidad en los programas de reducción de daño por tabaco y derivados del tabaco, también como herramienta para reducción y abandono del hábito tabáquico.

\section{CONCLUSIONES}

La poderosa industria del tabaco ha tomado al CE entre sus productos y quizá ésta sea una de las mayores amenazas porque puede aprovechar toda su maquinaria de promoción para tratar de colocarlo en todos los hogares. La regulación es indispensable; aunque de nuevo, el uso del CE se ha disparado por la lentitud de las instituciones y países para reaccionar. Varias de las organizaciones de enfermedades respiratorias han rechazado su uso, pero sobre todo debido a falta de regulación y de información de seguridad. Otros la han rechazado porque genera adicción a la nicotina, como si los cigarrillos convencionales disponibles a los menores a pesar de ser ilegal su acceso no lo hicieran, quitando una opción que parece menos tóxica a muchos fumadores que no pueden dejar de fumar por los métodos tradicionales.

\section{REFERENCIAS}

1. Bhatnagar A, Whitsel LP, Ribisl KM, et al.; American Heart Association Advocacy Coordinating Committee, Council on Cardiovascular and Stroke Nursing, Council on Clinical Cardiology, and Council on Quality of Care and Outcomes Research. Electronic cigarettes: a policy statement from the American Heart Association. Circulation 2014;130(16):1418-1436. doi: 10.1161/ CIR.0000000000000107.

2. Grana R, Benowitz N, Glantz SA. E-cigarettes: a scientific review. Circulation 2014;129(19):1972. doi: 10.1161/ CIRCULATIONAHA.114.007667.

3. Harrell PT, Simmons VN, Correa JB, Padhya TA, Brandon TH. Electronic nicotine delivery systems ("e-cigarettes"): review of safety and smoking cessation efficacy. Otolaryngol Head Neck Surg 2014;151(3):381-393. doi: 10.1177/0194599814536847.

4. McMillen RC, Gottlieb MA, Shaefer RM, Winickoff JP, Klein JD. Trends in Electronic Cigarette Use Among U.S. Adults: Use is Increasing in Both Smokers and Nonsmokers. Nicotine Tob Res 2014. pii: ntu213.

5. Centers for Disease Control and Prevention (CDC). Notes from the field: electronic cigarette use among middle and high school students - United States, 2011-2012. MMWR Morb Mortal Wkly Rep 2013;62(35):729-730.

6. Arrazola RA, Neff LJ, Kennedy SM, Holder-Hayes E, Jones $C D$; Centers for Disease Control and Prevention (CDC). Tobacco use among middle and high school students-United States, 2013. MMWR Morb Mortal Wkly Rep 2014;63(45):1021-1026. 
7. Johnston LD, O'Malley PM, Miech RA, et al. 2014 overview: Key findings on adolescent drug use. Monitoring the future: National survery results on drug use 1975-2014. Sponsored by The National Institute on Drug Abuse at The National Institutes of Health. Available from: http://www.monitoringthefuture.org/pubs/ monographs/mtf-overview2014.pdf

8. Hajek P, Etter JF, Benowitz N, Eissenberg T, McRobbie H. Electronic cigarettes: review of use, content, safety, effects on smokers and potential for harm and benefit. Addiction 2014;109(11):1801-1810. doi: 10.1111/ add.12659.

9. King BA, Patel R, Nguyen KH, Dube SR. Trends in awareness and use of electronic cigarettes among US adults, 2010-2013. Nicotine Tob Res 2015;17(2):219-227. doi: 10.1093/ntr/ntu191.

10. Dockrell M, Morrison R, Bauld L, McNeill A. E-cigarettes: prevalence and attitudes in Great Britain. Nicotine Tob Res 2013;15(10):1737-1744. doi: 10.1093/ntr/ntt057.

11. Collaco JM, Drummond MB, McGrath-Morrow SA. Electronic cigarette use and exposure in the pediatric population. JAMA Pediatr 2015;169(2):177-182. doi: 10.1001/jamapediatrics.2014.2898.

12. Niaura RS, Glynn TJ, Abrams DB. Youth experimentation with e-cigarettes: another interpretation of the data. JAMA 2014;312(6):641-642. doi: 10.1001/ jama.2014.6894.

13. Kandel ER, Kandel DB. Shattuck lecture. A molecular basis for nicotine as a gateway drug. N Engl J Med 2014;371(10):932-943. doi: 10.1056/NEJMsa1405092.

14. Bunnell RE, Agaku IT, Arrazola RA, et al. Intentions to smoke cigarettes among never-smoking US middle and high school electronic cigarette users: National Youth Tobacco Survey, 2011-2013. Nicotine Tob Res 2015;17(2):228-235. doi: 10.1093/ntr/ntu166.

15. Vardavas Cl, Filippidis FT, Agaku IT. Determinants and prevalence of e-cigarette use throughout the European Union: a secondary analysis of 26566 youth and adults from 27 Countries. Tob Control 2014. pii: tobaccocontrol-2013-051394. doi: 10.1136/tobaccocontrol-2013-051394.

16. Adkison SE, O'Connor RJ, Bansal-Travers M, et al. Electronic nicotine delivery systems: international tobacco control four-country survey. Am J Prev Med 2013;44(3):207-215. doi: 10.1016/j. amepre.2012.10.018.

17. Zhu SH, Gamst A, Lee M, Cummins S, Yin L, Zoref $\mathrm{L}$. The use and perception of electronic cigarettes and snus among the U.S. population. PLoS One 2013;8(10):e79332. doi: 10.1371/journal.pone.0079332.

18. Westenberger BJ. Evaluation of e-cigarettes. Available from: www.fda.gov/downloads/drugs/scienceresearch/ ucm173250.pdf

19. Trehy ML, Ye W, Hadwiger ME, et al. Analysis of electronic cigarette cartridges, refill solutions, and smoke for nicotine and nicotine related impurities. J Liq Chromatogr Relat Technol 2011;34:1442-1458.

20. Goniewicz ML, Knysak J, Gawron M, et al. Levels of selected carcinogens and toxicants in vapour from electronic cigarettes. Tob Control 2014;23(2):133-139. doi: 10.1136/tobaccocontrol-2012-050859.

21. Williams M, Villarreal A, Bozhilov K, Lin S, Talbot P. Metal and silicate particles including nanoparticles are present in electronic cigarette cartomizer fluid and aerosol. PLoS One 2013;8(3):e57987. doi: 10.1371/ journal.pone.0057987.

22. Kim HJ, Shin HS. Determination of tobacco-specific nitrosamines in replacement liquids of electronic cigarettes by liquid chromatography-tandem mass spectrometry. J Chromatogr A 2013;1291:48-55. doi: 10.1016/j.chroma.2013.03.035

23. Schraufnagel DE, Blasi F, Drummond MB, et al.; Forum of International Respiratory Societies. Electronic cigarettes. A position statement of the forum of international respiratory societies. Am J Respir Crit Care Med 2014;190(6):611-618. doi: 10.1164/rccm.201407-1198PP.

24. Cheng T. Chemical evaluation of electronic cigarettes. Tob Control 2014;23 Suppl 2:ii11. doi: 10.1136/tobaccocontrol-2013-051482.

25. Cameron JM, Howell DN, White JR, Andrenyak DM, Layton ME, Roll JM. Variable and potentially fatal amounts of nicotine in e-cigarette nicotine solutions. Tob Control 2014;23(1):77-78. doi: 10.1136/tobaccocontrol-2012-050604.

26. Vansickel AR, Eissenberg T. Electronic cigarettes: effective nicotine delivery after acute administration. Nicotine Tob Res 2013;15(1):267-270. doi: 10.1093/ntr/ ntr316.

27. Pellegrino RM, Tinghino B, Mangiaracina G, et al. Electronic cigarettes: an evaluation of exposure to chemicals and fine particulate matter (PM). Ann Ig 2012;24(4):279-288.

28. Hutzler C, Paschke M, Kruschinski S, Henkler F, Hahn J, Luch A. Chemical hazards present in liquids and vapors of electronic cigarettes. Arch Toxicol 2014;88(7):12951308. doi: 10.1007/s00204-014-1294-7.

29. Palazzolo DL. Electronic cigarettes and vaping: a new challenge in clinical medicine and public health. A literature review. Front Public Health 2013;1:56. doi: 10.3389/fpubh.2013.00056.

30. McAuley TR, Hopke PK, Zhao J, Babaian S. Comparison of the effects of e-cigarette vapor and cigarette smoke on indoor air quality. Inhal Toxicol 2012;24(12):850-857. doi: 10.3109/08958378.2012.724728.

31. Pisinger C, Døssing M. A systematic review of health effects of electronic cigarettes. Prev Med 2014;69:248. doi: 10.1016/j.ypmed.2014.10.009.

32. Farsalinos KE, Romagna G, Allifranchini E, et al. Comparison of the cytotoxic potential of cigarette smoke and electronic cigarette vapour extract on cultured myocardial cells. Int J Environ Res Public Health 2013;10(10):5146-5162. doi: 10.3390/ijerph10105146.

33. Bahl V, Lin S, Xu N, Davis B, Wang YH, Talbot P. Comparison of electronic cigarette refill fluid cytotoxicity using embryonic and adult models. Reprod Toxicol 2012;34(4):529-537. doi: 10.1016/j.reprotox.2012.08.001.

34. Behar RZ, Davis B, Wang Y, Bahl V, Lin S, Talbot P. Identification of toxicants in cinnamon-flavored electronic 
cigarette refill fluids. Toxicol In Vitro 2014;28(2):198208.

35. Vardavas Cl, Anagnostopoulos N, Kougias M, Evangelopoulou V, Connolly GN, Behrakis PK. Short-term pulmonary effects of using an electronic cigarette: impact on respiratory flow resistance, impedance, and exhaled nitric oxide. Chest 2012;141(6):1400-1406. doi: 10.1378/chest.11-2443.

36. Chatham-Stephens K, Law R, Taylor E, et al.; Centers for Disease Control and Prevention (CDC). Notes from the field: calls to poison centers for exposures to electronic cigarettes--United States, September 2010-February 2014. MMWR Morb Mortal Wkly Rep 2014;63(13):292293.

37. Jiménez RCA, Solano RS, de Granda OJl, et al. El cigarrillo electrónico. Declaración oficial de la Sociedad Española de Neumología y Cirugía Torácica (SEPAR) sobre la eficacia, seguridad y regulación de los cigarrillos electrónicos. Arch Bronconeumol 2014;50(8):362-367. doi: 10.1016/j. arbres.2014.02.006

38. Electronic nicotine delivery systems. Report by WHO. July 1, 2014. Access date: 2015 May 27. Available from: http://apps.who.int/gb/fctc/PDF/cop6/FCTC_COP6_10en.pdf?ua=1.

39. OMS. Cigarrillos electrónicos o sistemas electrónicos de administración de nicotina. Fecha de consulta: 22 de junio, 2015. Disponible en: (http://www.who.int/ tobacco/communications/statements/eletronic_cigarettes/es/)

40. Deeming tobacco products to be subject to the federal Food, Drug, and Cosmetic Act, as amended by the Family Smoking Prevention and Tobacco Control Act: regulations on the sale and distribution of tobacco products and required warning statements for tobacco products: proposed rules. Fed Regist 2014;79:23142-23207.

41. Marynak K, Holmes CB, King BA, Promoff G, Bunnell R, McAfee T; Centers for Disease Control and Prevention (CDC). State laws prohibiting sales to minors and indoor use of electronic nicotine delivery systems--United
States, November 2014. MMWR Morb Mortal Wkly Rep 2014;63(49):1145-1150.

42. Tobacco Control Legal Consortium. Deeming Regulation: What is possible under the law (Fact Sheet). Access date: 2015 May 27. Available from: publichealthlawcenter.org/sites/default/files/resources/tclc-fs-deeming-regwhat-is-possible-2014.pdf

43. Tobacco Control Legal Consortium. The FDA takes small steps to expand tobacco regulation (Fact Sheet). Access date: 2015 May 27. Available from: publichealthlawcenter.org/sites/default/files/resources/tclc-fs-fdatakesmallstepexpandtobaccoregulation-2014.pdf

44. Cámara de Senadores de los Estados Unidos Mexicanos. Ley General para el Control del Tabaco 2008; Gaceta Oficial del Senado de la República 198:115-23. Fecha de consulta: 6 de junio, 2015. Disponible en: http:// regulacionsanitarianl.gob. $\mathrm{mx} /$ node/360

45. Zhu SH, Sun JY, Bonnevie E, et al. Four hundred and sixty brands of e-cigarettes and counting: implications for product regulation. Tob Control 2014;23 Suppl 3:iii3-9. doi: 10.1136/tobaccocontrol-2014-051670.

46. McRobbie H, Bullen C, Hartmann-Boyce J, Hajek P. Electronic cigarettes for smoking cessation and reduction. Cochrane Database Syst Rev 2014;12:CD010216. doi: 10.1002/14651858.CD010216.pub2.

47. Rahman MA, Hann N, Wilson A, Mnatzaganian G, Worral-Carter L. E-cigarettes and smoking cessation: evidence from a systematic review and meta-analysis. PLoS One 2015;10(3):e0122544. doi: 10.1371/journal. pone. 0122544

\section{$\triangle$ Correspondencia:}

Dr. Rogelio Pérez Padilla

Instituto Nacional de Enfermedades Respiratorias Ismael Cosío Villegas. Calzada de Tlalpan Núm. 4502, Colonia Sección XVI, 14080, México, D.F. Correo electrónico: perezpad@gmail.com

Los autores declaran no tener conflicto de intereses. 